\title{
The possibility of self-assembly of complex nanostructures with pre-defined geometry under the action of laser field
}

\author{
Victoria S. Kornienko ${ }^{1,2, a)}$, Aleksey S.Tsipotan ${ }^{1}$, \\ Aleksandr S. Aleksandrovsky ${ }^{1,3}$, Vitaliy V. Slabko $^{1}$ \\ ${ }^{1}$ Siberian Federal University, Krasnoyarsk, Russia \\ ${ }^{2}$ Department of Computational Mathematics, Institute of Computational Modeling of Federal Research \\ Center KSC SB RAS, Krasnoyarsk, Russia \\ ${ }^{3}$ Laboratory of Coherent Optics, Kirensky Institute of Physics Federal Research Center KSC SB RAS, \\ Krasnoyarsk, Russia \\ ${ }^{a)}$ Corresponding author: vika-svetlakova@yandex.ru
}

\begin{abstract}
Self-assembly remains one of the simplest and cheapest methods of nanostructuring. And the dependence of the properties of the objects obtained, not only on their composition, but also on the form, brings to the fore the question of developing methods for forming structures of a predetermined form and searching for system parameters in which the formation of a structure becomes possible. This paper is devoted to modeling the process of self-assembly of a multiparticle nanostructure of a predetermined shape in the field of laser radiation.
\end{abstract}

\section{INTRODUCTION}

During recent decades, materials produced via the nanotechnology find applications in different branches of science and technology like electronics, optical communications, biology, etc [1-3]. Specifically, such nanoobjects like quantum dots and nanowires are widely used in solar panels [4], displays [5], multifunctional sensors [6] etc. The variability of optical properties of quantum dots in dependence of the size and shape allows modifying the properties of macroobjects based on them [7]. Therefore, controllable formation of nanostructures, especially, at the scale below diffraction limit of the light, is a persistent problem. Thereby, in recent decade's great attention of researches is devoted to the production [8-10], investigation of properties [11-12] and applications of new nanoparticles and the structures on their base [13]. Computer simulation of the processes of nanostructures' formation [11,14] allows optimizing the parameters of the system and to increase the efficiency of the processes of their creation.

Development of areas of science and technology mentioned above largely depends on the possibility to control the processes of nanostructuring; therefore, the elaboration of new efficient methods of creation of structures with characteristic dimensions below one micron, allowing industrial scale of production. Investigation of possibilities of formation of nanostructures is one of actively developing trends in laser physics [3,15-16]. Advances in this trend is connected with the dependence of the properties of the formed structure on the parameters of laser radiation such as wavelength, pulse duration, field intensity. Modern laser systems allow controlling these parameters that may be used for creation of nanostructures with pre-defined properties. Among various prospective approaches used in the nanostructures formation, more and more importance is gained by nanotechnologies utilizing self-organization. Selfassembly principle is that particles always tend to the lowest energy level in any system that leads to their reorientation and aggregation. The processes of spontaneous organization leading to formation of nanostructures often are attractive due to their reliability and low cost. 
Earlier in [17] a dynamical model was proposed for step-by-step self-assembly of nanoparticles in the field of laser radiation, in order to investigate the formation possibility of the structure consisting of three particles, and two cases were considered: with the participation of preliminarily formed spatially oriented pair and with the participation of arbitrarily oriented pair. Preliminary formation of pair requires additional efforts. Present study is aimed to investigation of the formation of stable nanostructures with pre-defined geometry from three initially arbitrarily positioned particles and the determination of parameters of the medium and the field enabling to talk on stability of formation process.

\section{THE MODEL OF NANOSTRUCTURES FORMATION}

The method of nanostructures formation based on self-organization under the action of quasi-resonant laser field is earlier proposed in [18-20]. The essence of this method is the inducing of the ac polarization on the individual nanoparticles and the proper choice of the parameters of the system ensuring the energy of the interaction between these nanoparticles would exceed the energy of the barrier preventing from spontaneous aggregation. Presence of optical resonances of the particles leads to the enhancement of interparticle interaction and, as well, is the basis for selective formation of different structures with pre-defined positions of particles within the nanostructure. Experiments on the formation of pairs composed of colloidal quantum dots under the action of laser radiation [21] demonstrated the possibility of the implementing of the proposed method.

When simulating the processes of interaction and assembly of nanoparticles, the description with the help of Lagevin equations system is widely used [12] that accounts for Brownian dynamics of an ensemble of particles :

$$
\left\{\begin{array}{l}
d \vec{r}_{j} / d t=\vec{V}_{j}, \\
m_{j}\left(d \vec{v}_{j} / d t\right)=\vec{F}_{j}-\vec{F}_{j}^{f}+\vec{F}_{j}^{c},
\end{array}\right.
$$

where $\vec{r}_{j}=\left(r_{j}^{x}, r_{j}^{y}, r_{j}^{z}\right)^{T}$ is the radius vector of the center of mass of $j$-th particle; $\vec{V}_{j}=\left(V_{j}^{x}, V_{j}^{y}, V_{j}^{z}\right)^{T}$ is the vector of translational velocity of the center of mass of $j$-th particle; $\vec{F}_{j}=-\operatorname{grad}\left(W_{j}^{v}+W_{j}^{e}+W_{j}^{d}\right), W_{j}^{v}$ is the Van der Waals energy, $W_{j}^{e}$ is the energy of electrostatic repulsion, $\vec{F}_{j}^{f}$ is the viscous friction force, and $\vec{F}_{j}^{c}$ is stochastic hydrodynamical force.

Let us suppose that the content of the medium is chosen so that electrostatic repulsion force exceeds Van der Waals force. This excess must be allow, at the same time, that switching on the laser radiation and, therefore, emerging attraction force induced by it may overrun electrostatic repulsion and formation of nanostructures held by Van der Waals force. Computer simulation was performed for CdTe quantum dots immersed into water solution. Let us choose the intensity of laser radiation to be $I=10^{6} \mathrm{~W} / \mathrm{cm}^{2}$ that corresponds to the intensity available by focusing the radiation of commercial Q-switched lasers. More detailed description of the computational model and of the choice pf medium parameters is given in [17].

The numerical solution of a system of ordinary differential equations (1) was obtained via two-step method of family of explicit numerical Runge-Kutta methods [22]. Calculation of particles motion was performed within a spherical cell initially filled by $N$ arbitrarily distributed particles. Radius of the cell was $20 \mathrm{~nm}$. At every iteration step for every particle, independently either it is isolated or involved into a Van der Waals nanostructure; the motion equations (1) are solved in accordance to the parameters described in [17].

The geometry of formed structure was controlled by the frequency and orientation of polarization vector of external field. The probability of structure formation with certain geometry is estimated statistically from large number of identical calculations. In the course of simulations the following stable structures were found: "line" (when third particle adjoins to a pair at the angle $0^{\circ} \pm 5^{\circ}$ from the line connecting the centers of mass of particles comprising the pair), "angle" $\left(90^{\circ} \pm 5^{\circ}\right)$, "pyramid" $\left(120^{\circ} \pm 5^{\circ}\right)$.

\section{POSSIBILITY OF FORMATION OF STRUCTURES WITH PRE-DEFINED SHAPE}

As already was noted above, the paper [17] proves the possibility of two-stage formation of multiparticle structure. Probability of the assembly per a single laser pulse in this case exceeded $40 \%$. However, with the increase 
of the number of particles in the structure to be formed the complexity of the implementation of step-to step assembly grows essentially. Therefore, the most interesting case is when all particles are initially isolated, and action of laser radiation would lead to their assembly into pre-defined multiparticle structure.

Lets consider the case when particles are initially positioned according to Fig. 1.

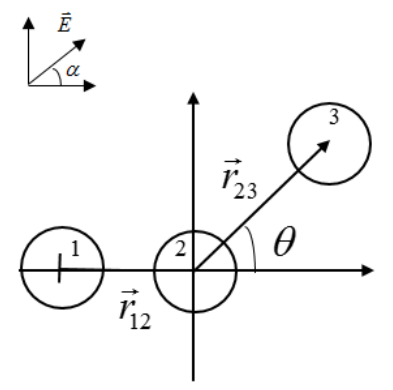

FIGURE 1. Initial positions of particles.

It is known that the energy of interparticle interaction in the field of laser radiation depends on the light intensity, on the interparticle distance, on the radiation frequency, on the resonant frequencies of the particles and on the orientation of their ensemble with respect to polarization plane of radiation. The potential well is formed in the spectral-angular dependence of dipole-dipole interaction energy, and if the depth of potential well exceeds the repulsion energy of particles, then the formation of stable structure becomes possible. Therefore, the first stage of simulation is the numerical calculation of the energy of interaction between the third particle and the particles 1 and 2 in dependence on wavelength and orientation of polarization vector of the external field. Let us take the distance between the first and the second particles $\left|\vec{r}_{12}\right|$ to be $12 \mathrm{~nm}$, and the distance between the second and the third particles $\left|\vec{r}_{23}\right|$ we will set to be 20,16 or $10 \mathrm{~nm}$, while the angle between vectors $\vec{r}_{12}$ and $\vec{r}_{23} \theta$ can be taken arbitrarily.

Calculated dependence of the energy of dipole-dipole interaction of all three particles normalized by the thermal energy $k T$ at room temperature on the external field wavelength and the angle $\alpha$ between polarization vector and $\vec{r}_{12}$ is plotted in Fig. $2 \vec{r}_{23}=16 \mathrm{~nm}: \theta=0^{\circ}$ (Fig, 2a), $\theta=120^{\circ}$ (Fig.2b).

Fig. 2 evidences that choice of the angle $\alpha$, defining the direction of polarization vector of external field as well as the choice of the wavelength and the choice of position of the third particle considerably influence the character of energy spectrum. So, for the angle $\theta=0^{\circ}$ at $\alpha=0^{\circ}$ and $\alpha=180^{\circ}$ (Fig. 2a) the potential well as deep as $-8 k T$ is observed in the region of $770 \mathrm{~nm}$, that means the possibility of formation of stable nanostructure. Spectral width of this potential well is of order $100 \mathrm{~nm}$. Different behavior of potential surface can be found for the angle $\theta=120^{\circ}$ where potential well of the depth $-10 k T$ and width $30 \mathrm{~nm}$ is observed at $\alpha=90^{\circ}$ (Fig, 2b). In this case, the potential well is short-wavelength-shifted and corresponds to the wavelength $440 \mathrm{~nm}$.

When the distance between particles decreases, the minimum of interaction energy shifts either to the longerwavelength part of the spectrum (Fig, 3a) or to the short-wavelength one (Fig. 3b) for the first or the second case, respectively. Thus, the wavelengths $\lambda^{r}=770 \mathrm{~nm}$ and $\lambda^{r}=440 \mathrm{~nm}$ enable maximum energies of attraction and can be chosen for the formation of nanostructure from a wandering triple of particles. It was supposed in these calculations that the third particle is positioned on the circle centered at the particle 2 with the radius $16 \mathrm{~nm}$, that corresponds to the volume concentration $n=0.0025$. 


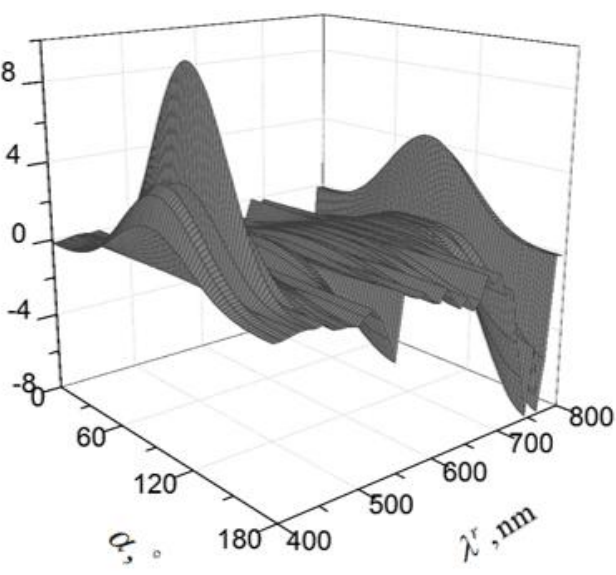

(a)

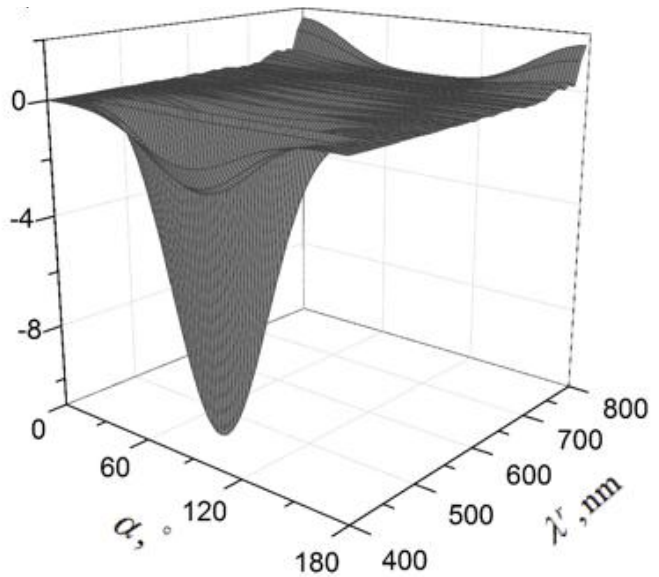

(b)

FIGURE 2. Dependence of dipole-dipole interaction energy for three nanoparticles on the angle $\alpha$ and the external field wavelength $\lambda^{r}, \vec{r}_{23}=16 \mathrm{~nm}, \theta=0^{\circ}$ (a), $\theta=120^{\circ}$ (b).

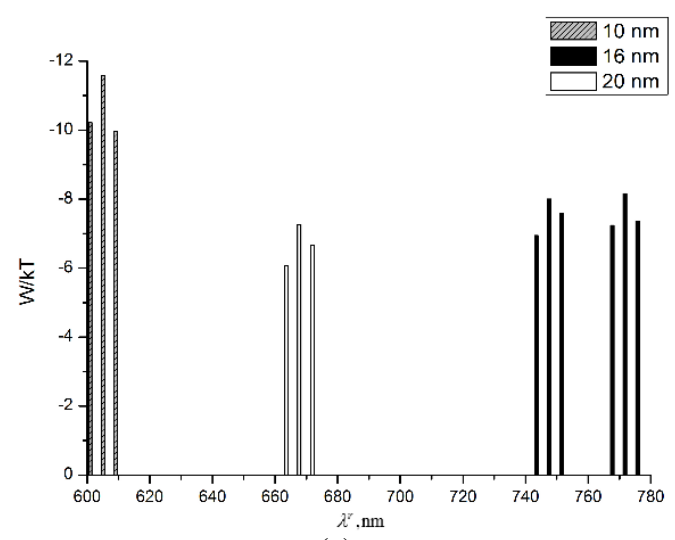

(a)

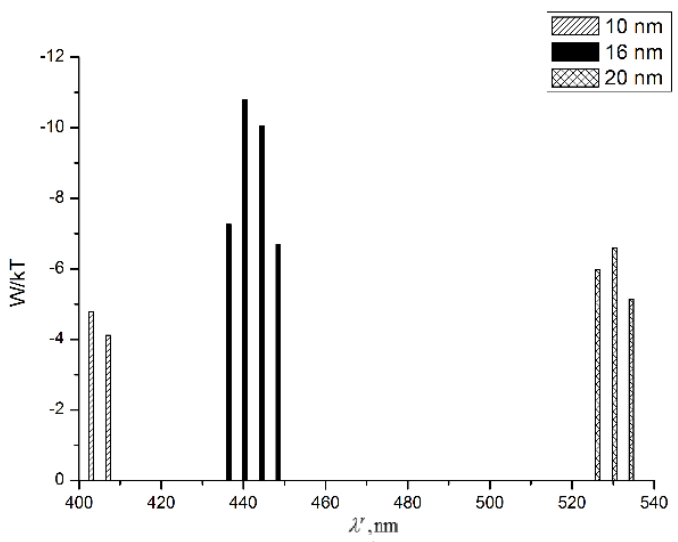

(b)

FIGURE 3. Minimum of dipole-dipole interaction energy in dependence on the external field wavelength for different distances between particles for $\theta=0^{\circ}, \alpha=0^{\circ}$ (3a) и $\theta=120^{\circ}, \alpha=90^{\circ}$ (3b)

Let us then estimate the probability of formation of nanostructures under optimal parameters chosen above. We sequentially take the wavelength of external field to be either $\lambda^{r}=770 \mathrm{~nm}$ or $\lambda^{r}=440 \mathrm{~nm}$ and will consider that particles 2 and 3 can be positioned at arbitrary points on a circles centered on the particle 1 with radii 12 and 16 $\mathrm{nm}$, respectively. Calculated probabilities of the assembly of the structures of three particles during the time period not exceeding $10 \mathrm{~ns}$ for $\lambda^{r}=770 \mathrm{~nm}$ and $\lambda^{r}=440 \mathrm{~nm}$ are plotted in Figs. 4 and 5 respectively. In addition, Figs. 4 and 5 show that the probability of assembling the structure is significantly reduced with deviating from the resonant wavelengths.

Maximum probability (11\%) for longer-wavelength external field case is found for the formation of linear structure at the mean assembly time $6.34 \mathrm{~ns}$. The orientation angle of external field polarization corresponding to maximum assembly equals to $0^{\circ}$, in good agreement with calculations of dipole-dipole interaction energy calculations (Fig.2a). 57\% of simulation runs did not terminated by the formation of a structure, and 32\% of simulation runs resulted in formation of structures other than linear one.

For the case of self-assembly induced in the shorter-wavelength range the structures of angle type and pyramid type are formed with the probability $8 \%$ and $10 \%$, respectively and $23 \%$ of simulation runs do not terminate by an assembly; the rest of runs resulted in formation of structures other than noted above. Mean time of assembly into pyramid is $5.18 \mathrm{~ns}$, and for the angle structure this time equals to $5.03 \mathrm{~ns}$. The external field polarization orientation angles corresponding to maximum probability of assembly are $45^{\circ}$ for angular structure and $90^{\circ}$ for pyramidal structure. 


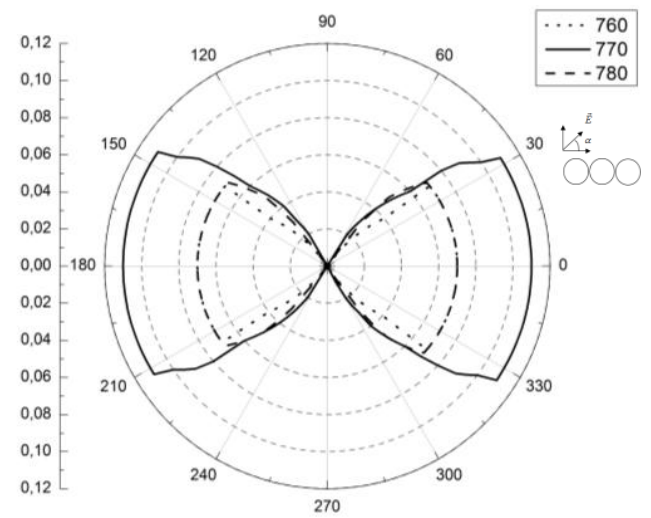

FIGURE 4. Probability of assembly of a triple of particles into linear structure in dependence on their mutual orientation at $\lambda^{r}=760,770,780 \mathrm{~nm}$

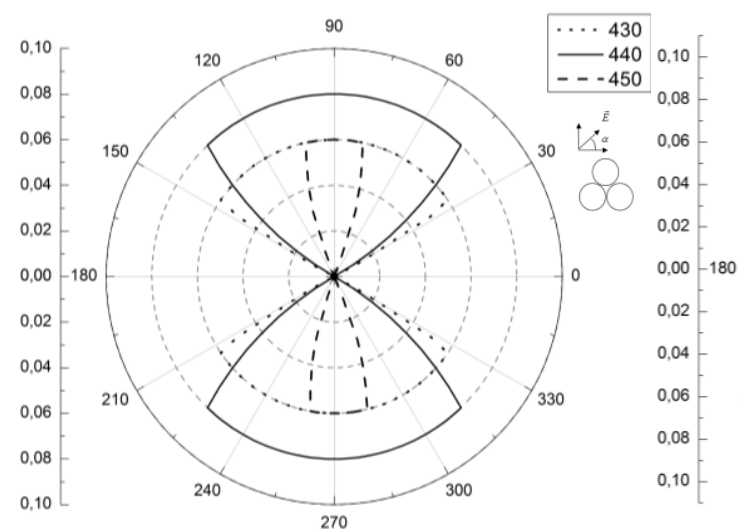

(a)

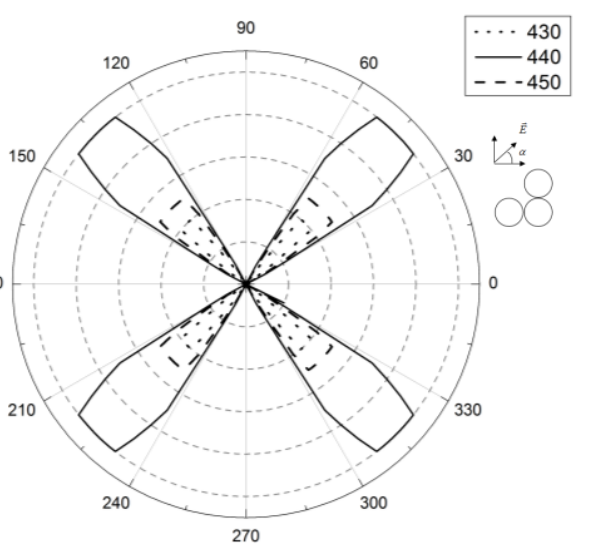

(b)

FIGURE 5. Probability of assembly of a triple of particles into pyramidal structure (a) and into angular structure (b) in dependence on their mutual orientation at $\lambda^{r}=430,440,450 \mathrm{~nm}$

Simulations show that under properly chosen external field wavelength and orientation of polarization vector with respect to positions of particles the self-assembly of different stable structures is possible. The probability of assembly of required structures per single pulse reaches values of order of $10 \%$ at modest intensity of external field, with no preliminary formation of a pair of particles.

\section{CONCLUSIONS}

In this paper the principal possibility of selective formation of structures with pre-defined geometry from thee nanoparticles is demonstrated in the field of laser radiation escaping step-by-step assembly. In this case, the probability of formation of pre-defined nanostructures reaches $10-11 \%$ during $10 \mathrm{~ns}$ laser pulse at the modest external field intensity. The possibility to control the geometry of formed structure by the choice of external field wavelength is also discussed. Note, that the source for inducing the self-assembly of the particles of arbitrary size must be tunable across the visible spectral range. This requirement is satisfied by commercial parametric oscillator pumped by Q-switched laser.

\section{ACKNOWLEDGMENTS}

The reported study was funded by Russian Science Foundation (Grant 18-72-00003). V.V. Slabko is grateful for the support from the Ministry of Education and Science of the Russian Federation (Grant 3.6341.2017/VU). 


\section{REFERENCES}

1. W. Paraket al., Nanotechnology, 14(7), R15-R27 (2003)

2. V. Salata, Journal of nanobiotechnology, 2,№3 (2004)

3. G. Cao, Nanostructures \& nanomaterials-Synthesis. Properties \& Application (Imperial College Press, London, 2008), pp.63-73.

4. W. Hu et al,Journal of Applied Physics, 114, 124509-124509 (2013)

5. M. Ganshari. Quantum-dot Based Light-emitting Diodes.(SpiGlobals, Tehran, 2017), 193 p.

6. Zh. Qian et al., Bioimaging Application, and Multifunctional Sensor, ACS Appl. Mater. Interfaces,6(9), 67976805(2014)

7. W. Lei, H. H. Tan, and C. Jagadish. Appl. Phys. Lett.95, 013108, (2009)

8. H. Caiyun, K. Xianggui, J. Meihong, and L. Xiaodong, Journal of Materials Science, 53(2),1123-1134 (2018)

9. H. Mohsen et.al., Applied Surface Science,439,1163-1179 (2018)

10. M. Khenner, S. Yadavali, R. Kalyanaraman, Phys Fluids,23,122105-122118(2011)

11. A. Isiaka, N. H. Mahouton. J. Phys. A: Math. Theor. 44,№465208 (2011)

12. S.P. Domingos, S.A. Salazar. J. Phys. A: Math. Theor.49, № 465001 (2016)

13. Y. Zhang, Z. Zhang, J. Guan.J. Phys. A: Math. Theor.40,12365-12375, (2007)

14. D.Grieser, J. Phys. A:Mathematical and General,42(13), 135204, (2009)

15. M. Khenner, S. Yadavali and R. Kalyanaraman, Mathematical Modeling of Natural Phenomena, 7, 20-38, (2012)

16. V. Simakin, A Voronov, and V. Shafeev,Phys. Wave Phenom.,15, 218-240 (2007)

17. V.S. Kornienko, A.S. Tsipotan, A.S. Aleksandrovsky and V.V. Slabko, Photonics and Nanostructures Fundamentals and Applications, 35, 100707(2019)

18. V. V. Slabko, G. G. Khachatryan, and A. S. Aleksandrovsky, JETP Lett. 84, 300-304 (2006).

19. V.V.Slabko, A.S.Tsipotan, A.S. Aleksandrovsky, Photonics and nanostructures-fundamentals and applications, 10, 636-643(2012).

20. V.V. Slabko, A.S. Tsipotan, A.S. Aleksandrovsky, E.A. Slyusareva. Applied Physics B, 117(1),271-278 (2014)

21. A.S. Tsipotan, M.A. Gerasimova, V.V. Slabko and A.S. Aleksandrovsky, Optics Express 24, 1114511150(2016).

22. J.C. Butcher. Numerical Methods for Ordinary Differential Equations (Wiley, New York, 2008), 468 p. 